\title{
Antipsychotikainduzierte tardive Syndrome
}

\author{
W. Wolfgang Fleischhacker · Alex Hofer · Christian Jagsch · Walter Pirker · Georg Psota · Hans Rittmannsberger • \\ Klaus Seppi
}

Eingegangen: 7. März 2016 / Angenommen: 14. Juni 2016 / Online publiziert: 31. August 2016

(c) Der/die Autor(en) 2016. Dieser Artikel ist eine Open-Access-Publikation.

Zusammenfassung Tardive Syndrome bleiben trotz des vermehrten Einsatzes von Antipsychotika der neuen Generation ein relevantes klinisches Problem. Es handelt sich um schwer behandelbare Bewegungsstörungen mit geringer Rückbildungstendenz. Deswegen ist die Prophylaxe von entscheidender Bedeutung, wobei der verantwortungsvolle Einsatz von Antipsychotika im Vordergrund steht. Für die Therapie der tardiven Dyskinesie gilt, auch wenn die Evidenzlage eingeschränkt ist, dass das Antipsychotikum, wenn möglich, abzusetzen ist. Wenn nicht, sollte eine Umstellung auf ein Antipsychotikum mit niedrigem Risiko für tardive Dyskinesien erfolgen. Die Evidenzlage und Zulassungssituation für andere symptomatische Therapien der tardiven Dyskinesie ist nach wie vor unbefriedigend.

Univ.-Prof. Dr. W. W. Fleischhacker $(\bowtie)$.

Assoz.-Prof. PD Dr. A. Hofer

Universitätsklinik für Psychiatrie I, Department für

Psychiatrie, Psychotherapie und Psychosomatik,

Medizinische Universität Innsbruck,

Anichstraße 35, 6020 Innsbruck, Österreich

wolfgang.fleischhacker@i-med.ac.at

Prim. Dr. C. Jagsch

Abteilung für Alterspsychiatrie und Alterspsychotherapie,

LKH Graz Süd-West, Graz, Österreich

Univ.-Prof. Dr. W. Pirker

Wilhelminenspital, Wien, Österreich

Prim. Dr. G. Psota

Psychosozialer Dienst, Wien, Österreich

Prim. Univ.-Prof. Dr. H. Rittmannsberger

Psychiatrische Abteilung 1, Landes-Nervenklinik

Wagner-Jauregg, Linz, Österreich

Univ.-Prof. Dr. K. Seppi

Universitätsklinik für Neurologie, Medizinische Universität Innsbruck, Innsbruck, Österreich
In diesem Manuskript ist der aktuelle Wissensstand zu Phänomenologie, Verlauf, Prävention und Therapie von tardiven Dyskinesien überblicksweise zusammengefasst.

Schlüsselwörter Tardive Syndrome - Tardive Dyskinesie $\cdot$ Antipsychotika $\cdot$ Prophylaxe $\cdot$ Therapie

\section{Antipsychotic-induced tardive syndromes}

Summary Tardive dyskinesia (TD) remains a relevant clinical problem despite the increasing use of newgeneration antipsychotics. Antipsychotic-induced tardive syndromes are difficult to treat and have a low tendency of remission. Therefore, prophylaxis is of utmost importance, with the responsible use of antipsychotics as a prime desideratum. With respect to managing tardive dyskinesia, discontinuing the antipsychotic, if possible, albeit not backed up by unequivocal evidence, is still the main recommendation. If this is not possible, the switch to an antipsychotic with a lower TD risk is the next-preferred option. Other symptomatic treatments have been explored, but clinical trials have provided inhomogeneous results and only very few compounds are approved for the treatment of tardive dyskinesia. This manuscript summarizes the current evidence with respect to the phenomenology, course, prevention and treatment of tardive syndromes.

Keywords Tardive syndrome $\cdot$ Tardive dyskinesia $\cdot$ Antipsychotic $\cdot$ Prophylaxis · Treatment

\section{Einleitung}

Antipsychotika sind der Goldstandard in der Behandlung von Schizophrenie sowie akuter Manie und finden auch im Management von nichtpsychotischen 
Störungen und Verhaltensauffälligkeiten bei Demenz häufig im Off-Label-Bereich breite Anwendung. Eine der Möglichkeiten, die Gruppe der Antipsychotika zu unterteilen, ist entsprechend ihrer Markteinführung in Antipsychotika der ersten Generation (First Generation Antipsychotic Drug [FGA]) ${ }^{1}$ und der neueren Generation (New Generation Antipsychotic Drug $[\mathrm{NGA}])^{2}$.

Schon bald nach Einführung der Neuroleptika, wie FGA damals genannt wurden, war klar, dass diese, wie alle Dopaminrezeptor-Antagonisten (DRA), mit einem erhöhten Risiko für extrapyramidal-motorische Nebenwirkungen vergesellschaftet sind. Tardive Dyskinesien (TD) zählen zu den schwerwiegendsten motorischen Nebenwirkungen von DRA, da sie häufig irreversibel sind und zu permanenter Behinderung führen können [1]. Mit Einführung der NGA konnte das Risiko für extrapyramidal-motorische Symptome (EPMS) zwar deutlich reduziert werden, allerdings treten EPMS auch bei der Anwendung von NGA bei einer nicht zu vernachlässigenden Zahl von Patienten auf [2].

\section{Definition}

Der Begriff „tardiv“ leitet sich historisch aus dem französischen ab (,tardif") und bedeutet langsam bzw. verzögert, während der Begriff „Dyskinesie“ (griechisch: $d y s=$ miss sowie kinesis = Bewegung) abnorme bzw. gestörte Bewegung bedeutet. Spätdyskinesien wurden ursprünglich von Sigwald et al. als „dyskinesie facio-bucco-linguo-masticatice“ beschrieben [3], der Terminus „tardive dyskinesia“ findet sich zum ersten Mal bei Faurbye et al. [4].

Gemäß DSM-5 inkludiert das Spektrum der TD unwillkürliche Bewegungen der Zunge, des Kiefers, der Extremitäten oder des Rumpfes [5]. Sie können athetoid oder choreiform sein. Die Bewegungsstörungen treten nach der DRA-Exposition innerhalb von mindestens einigen Monaten auf und können nach Absetzen der Medikation persistieren. Bei älteren Patienten können sich die Symptome auch schon nach kürzerer Medikationsdauer entwickeln.

\section{Phänomenologie}

Unter dem Überbegriff TD wurden unterschiedliche Bewegungsstörungen beschrieben. In jüngerer Zeit besteht die Tendenz, den Begriff „klassische TD“ für orobuccolinguale Dyskinesien zu reservieren und eine spezifischere Terminologie, die auf der Phänomenologie der einzelnen Subtypen des tardiven Syndroms (TS) basiert, zu verwenden [6]. Dazu zählen TD, tar-

\footnotetext{
1 FGA: First Generation Antipsychotic Drug, typische Antipsychotika (z. B. Haloperidol)

2 NGA: New Generation Antipsychotic Drug, atypische Antipsychotika
}

dive Dystonie, tardiver Tremor, tardive Akathisie, tardiver Myoklonus sowie tardiver Tourettismus.

Häufig ähneln die Charakteristika der TD normalen Bewegungsmustern wie Kauen, Verziehen der Mundwinkel, zugreifende Bewegungen oder „Klavierspielen“, die jedoch unwillkürlich durchgeführt werden [7]. In vielen Fällen sind sich die Patienten dieser Bewegungen nicht bewusst.

In der Regel ist bei der klassischen TD die Gesichtsmuskulatur, insbesondere die orale und periorale Muskulatur, betroffen [8]. Die Dyskinesie der Zunge ist durch langsame und repetitive Bewegungen in der Mundhöhle charakterisiert, wodurch die Wangen ausgebeult werden („Bonbonzeichen“). Grimassieren, Anheben der Augenbrauen oder Stirnrunzeln können ebenfalls Zeichen der klassischen TD sein.

Viele Patienten beugen und strecken einzelne Finger ähnlich wie beim Gitarre- oder Klavierspielen. Dyskinesien der Beine können den Eindruck langsamen Tretens auf der Stelle erwecken. Dyskinesien des Zwerchfells führen zu abgehackter Sprache oder unkontrollierbarem Stöhnen.

Der Begriff „tardive Dystonie“ beschreibt unwillkürliche, dystone Bewegungen. Dystonie ist als anhaltende, unwillkürliche Muskelaktion definiert, die zu wiederholten Bewegungen oder abnormen Haltungen häufig mit drehender Komponente führt. Oft existiert aber das Phänomen einer "geste antagoniste“. Das bedeutet, dass Patienten häufig in der Lage sind, die Dystonie durch einfache taktile Manöver aufzuheben, etwa durch das Berühren des Kinns mit dem Finger einen Torticollis zu lösen [9]. Aus bislang nicht bekannten Gründen scheinen Männer besonders suszeptibel für tardive Dystonien zu sein [10].

\section{Epidemiologie}

Die tatsächliche Prävalenz der TD lässt sich nur schwer abschätzen. So sind sich viele Patienten, insbesondere jene mit klassischer, buccolinguomastikatorischer (BLM) Trias, ihrer Symptome nicht bewusst. Die Bewegungsstörung kann über die Zeit fluktuierend und die Symptomatik kann durch die Medikation maskiert sein [6]. Die klassische TD ist die häufigste persistierende Nebenwirkung nach langem Antipsychotikagebrauch. Schätzungen zufolge liegt die Gesamtprävalenz der TD bei mit Antipsychotika behandelten, ambulanten Patienten bei rund $30 \%$. Die Inzidenz wurde auf 4-8\% mit einer kumulativen 5-Jahres-Inzidenz von $25 \%$ geschätzt, wobei bei rund $2 \%$ pro Jahr eine Spontanremission zu beobachten ist [11].

\section{Verlauf}

Die TD beginnt definitionsgemäß einige Monate bis mehrere Jahre nach Einleitung einer Antipsychotikatherapie. Typischerweise beginnt die TD schleichend, erreicht rasch ihren Höhepunkt und stabilisiert sich 
dann, wobei es zu fluktuierenden Verläufen mit wechselnder Ausprägung der Symptome kommen kann. Eine Verschlechterung nach einer Phase der Stabilisierung ist unüblich. Bei einem Großteil der Patienten bestehen die Symptome der TD auch noch 5 Jahre nach Absetzen der antipsychotischen Therapie [12, 13].

So hat eine Langzeitbeobachtung an Patienten unter Therapie mit FGA über 10 Jahre gezeigt, dass die TD sich über den gesamten Zeitraum nur unwesentlich verändert hat und es zu keiner dramatischen Verschlechterung der Symptomatik gekommen ist [14].

\section{Risikofaktoren}

Höheres Alter, die Dauer und kumulative Gesamtdosis der kontinuierlichen Therapie mit Antipsychotika sind Risikofaktoren, die das Auftreten von TD begünstigen. Unter den jüngeren Patienten tragen Männer und unter den älteren Patienten Frauen ein höheres TD-Risiko [15]. Möglicherweise ist das Auftreten eines antipsychotikainduzierten Parkinsonsyndroms in der Anamnese ebenfalls ein Risikofaktor. Eine Sensitivität des extrapyramidalen Systems scheint eine Voraussetzung für das Auftreten von TD zu sein [16].

FGA sind mit einem höheren TD-Risiko assoziiert als NGA [17-19]. Dies könnte darauf zurückzuführen sein, dass NGA eine im Vergleich zu Dopamin stärkere Serotoninrezeptorblockade aufweisen und diese starken serotonergen Blockaden mit weniger EPMS assoziiert sind [20]. Darüber hinaus werden auch Zusammenhänge zwischen TD, afroamerikanischer Herkunft, dem Vorliegen affektiver sowie organischpsychiatrischer Störungen, Diabetes sowie Alkoholund Kokainkonsum in der Anamnese beschrieben [21].

Unterschiede bezüglich des Auftretens der TD bestehen auch hinsichtlich der verabreichten Substanzklasse. So hat ein systematisches review, in das die Daten von 30.139 Patienten aus 12 Langzeit- und 4 Querschnittstudien eingeflossen sind, gezeigt, dass die Inzidenz von TD bei Anwendung von NGA im Vergleich zu FGA signifikant geringer ist [22]. Demnach lag die jährliche Inzidenz der TD in der Gruppe der Erwachsenen bei $3 \%$ bei NGA vs. $7,7 \%$ bei FGA $(p<0,0001)$. Die Inzidenz von TD bei mit NGA behandelten Kindern und Jugendlichen war mit 0,4\% gering. Die Autoren dieses Reviews machen allerdings darauf aufmerksam, dass die kürzere Einnahmedauer und die geringeren Dosierungen für das seltene Auftreten von TD in dieser Altersgruppe verantwortlich sein könnten.

Auf mögliche genetische Risikofaktoren wird im Kapitel „Pathophysiologie“ hingewiesen.

\section{Differenzialdiagnose}

Obwohl die Antipsychotikabehandlung häufig die Ursache von Bewegungsstörungen bei psychiatrischen
Patienten ist, müssen immer auch andere Ursachen in Betracht gezogen werden, da Bewegungsstörungen auch Zeichen neurologischer und anderer Erkrankungen sein können. So können sich zahlreiche neurologische Erkrankungen, wie bspw. Morbus Huntington oder Morbus Wilson initial durch isolierte neuropsychiatrische Symptome manifestieren, die dem Beginn motorischer Störungen viele Jahre vorausgehen können [7].

Es existieren keine definitiven klinischen, elektrophysiologischen oder Laborparameter, um TD zu diagnostizieren. Einige Zeichen geben, wenn sie vorhanden sind, Hinweise auf das Vorliegen einer anderen Diagnose als TD [7]:

- rasche Verschlechterung,

- konstant unilaterale Manifestation,

- Vorhandensein zusätzlicher neurologischer Symptome wie Demenz, sensorische Störungen oder Harninkontinenz,

- Auftreten zusätzlicher somatischer Symptome wie Ikterus, Fieber, Hautrötung etc.

Untypische Verläufe, die nicht in das Bild einer TD passen, sollten an einer Spezialabteilung abgeklärt werden.

\section{Morbus Huntington}

Die Huntington-Krankheit ist eine differenzialdiagnostische Herausforderung, da die Bewegungsstörungen bei beiden Erkrankungen phänomenologisch identisch erscheinen können und bei beiden Diagnosen neuropsychiatrische Symptome den Bewegungsstörungen vorausgehen können. Bei sorgfältiger Beobachtung lassen sich beim M. Huntington jedoch typische motorische Muster erkennen. So sind die choreatischen Überbewegungen zufällig von einer auf die andere Körperregion fließend. Häufig werden sie von willkürlichen Bewegungen, die die unfreiwilligen Bewegungsmuster kaschieren sollen, maskiert. Bei der TD sind die Bewegungen repetitiv („repetitive Chorea“) und betreffen bevorzugt die orofaziale Region. Die obere Gesichtshälfte ist bei TD seltener betroffen als bei M. Huntington. Typische klinisch-neurologische Zeichen eines M. Huntington sind dagegen die motorische Impersistenz (z. B. die Unfähigkeit, die Zunge länger als $10 \mathrm{~s}$ aus dem Mund zu strecken), Optomotorikstörungen (verlangsamte Sakkadeninitiation und -geschwindigkeit) und Parkinsonsymptome bzw. motorische Ungeschicklichkeit, die häufig stärker behindern als die Chorea. Daneben besteht bei bis zu einem Viertel der neu diagnostizierten Huntington-Patienten eine negative Familienanamnese, weshalb im Zweifelsfall nach Aufklärungsgespräch und schriftlicher Einwilligung eine genetische Testung zum Nachweis oder Ausschluss eines M. Huntington zu empfehlen ist. 


\section{Morbus Wilson}

Obwohl die Dystonie bei beiden Erkrankungen häufig die kraniozervikale Region betrifft, deuten Retrocollis oder axiale Dystonien eher auf TD hin, während oromandibuläre Dystonien häufiger bei M. Wilson auftreten. Morbus Wilson ist eine autosomal-rezessiv vererbte Erkrankung mit einer Vielzahl von Symptomen, die von psychiatrischen Störungen bis hin zu Bewegungsstörungen reichen.

An M. Wilson ist zu denken, wenn ein Jugendlicher oder Erwachsener unter 50 Jahren eine Erhöhung der Leberenzyme, Dysphagie oder Dysarthrie, die nicht durch eine neurologische Erkrankung erklärt werden können, Bewegungsstörungen sowie psychiatrische Symptome aufweist. Darüber hinaus ist bei Jugendlichen mit affektiven Psychosen und gering erhöhten Lebertransaminasen, bei Patienten mit Coombs-negativer hämolytischer Anämie sowie bei Patienten mit unerklärbarer Leberzirrhose und Leberversagen das Vorliegen eines M. Wilson in Erwägung zu ziehen. Die effektivsten Screeningmaßnahmen sind die Spaltlampenuntersuchung (Kayser-Fleischer-Ring) und die Bestimmung von Kupfer im 24-Stunden-Harn. Daneben haben beinahe alle Neuro-Wilson-Patienten auch MRI-Veränderungen im Bereich der Basalganglien oder im Mittelhirn.

\section{Absetzphänomene (,withdrawal emergent syndrome")}

Absetzphänomene können TD-Manifestationen bei Erwachsenen stark ähneln [23]. Diese Dyskinesien treten nach abruptem Absetzen von Antipsychotika nach lang andauerndem Gebrauch auf. Im Gegensatz zur klassischen TD betreffen die Hyperkinesien primär die Gliedmaßen, den Hals und den Rumpf. Der untere Gesichtsbereich ist seltener betroffen. Dyskinesien im Rahmen der Absetzphänomene treten üblicherweise einige Tage nach Absetzen der antipsychotischen Medikation auf. Der Verlauf ist kürzer und benigner als bei TD. Die Dyskinesie verschwindet typischerweise innerhalb von 3 Monaten.

\section{Pathophysiologie}

Zur Pathophysiologie der TD gibt es eine Reihe von Hypothesen. Die am weitesten verbreitete Hypothese ist die Theorie der Dopaminrezeptorhypersensitivität [24-27]. Demnach führt der chronische Gebrauch von DRA zu einer Sensibilisierung der Dopaminrezeptoren, was in einem Überschießen der dopaminergen Neurotransmission im nigrostriatalen System und damit im Verlust der Bewegungskontrolle resultiert. Während die direkte Evidenz für diese Hypothese im Tiermodell sehr stark ist, konnte deren Gültigkeit beim Menschen nicht bestätigt werden.

Eine alternative Hypothese besagt, dass die TD ein Resultat der Neurodegeneration von striatalen Inter- neuronen aufgrund von oxidativem Stress ist [28-30]. Der oxidative Stress könnte dabei Resultat der erhöhten Produktion von freien Radikalen aufgrund des durch lange andauernden Antipsychotikagebrauch ausgelösten erhöhten Dopaminumsatzes sein.

Genetische Assoziationsstudien zeigen, dass verschiedene genetische Variationen bei TD den Metabolismus von Medikamenten etwa über das CYP450-, CYP2D6- oder CYP1A2-System beeinflussen [31-33]. Kleinere Studien deuten auf die Beteiligung von Genen hin, die die Neurotransmission und die synaptische Plastizität regulieren. Diese synaptische Plastizität könnte maladaptiv sein [34].

Andere Forschungsgruppen haben postuliert, dass bei Patienten mit TD bestimmte Subgruppen von striatalen GABAergen Interneuronen geschädigt oder dysfunktional sind $[35,36]$. Eine Übersicht zur Pathophysiologie tardiver Syndrome findet sich in dem rezenten Review von Cloud et al. [37].

\section{Prävention der tardiven Dyskinesie}

Da es sich bei der TD definitionsgemäß um eine iatrogene Störung handelt, kommt der Prävention eine entscheidende Rolle zu. Sind erste Anzeichen einer TD aufgetreten, so ist, wenn möglich, ein Absetzen der antipsychotischen Medikation ernsthaft in Erwägung zu ziehen [7]. Frühes Erkennen und ggf. Absetzen des auslösenden Medikaments, insbesondere bei jüngeren Patienten, bieten die beste Chance für Remission [38].

\section{Verzicht auf Antipsychotika}

Primäre Maßnahme zur Prävention von TD wäre die Vermeidung von Antipsychotika, so dies möglich ist. Wenn eine Therapie mit einem Antipsychotikum unumgänglich ist, so ist bei der Indikationsstellung besondere Sorgfalt geboten. Off-Label-Gebrauch von Antipsychotika sollte nur nach sorgfältiger Prüfung auf Basis der geltenden gesetzlichen Regelungen erfolgen [39]. Gemäß der Deklaration von Helsinki der WMA (World Medical Association) können „[...] im Rahmen der Behandlung eines Patienten unerprobte Behandlungen durch den Arzt eingesetzt werden, wenn keine bekannte Behandlungsform existiert oder diese ineffektiv war und das qualifizierte Einverständnis des Patienten vorliegt" [40].

Da Patienten mit akut auftretenden EPMS unter Antipsychotikatherapie auch ein erhöhtes Risiko für TD haben [41], ist auf deren frühe Anzeichen zu achten. Dies gilt auch für Patienten mit anderen Risikofaktoren für TD. Patienten, Betreuungspersonen und Angehörige sollten über das Risiko des Auftretens von EPMS inklusive TD und die Frühsymptomatik von TD informiert und zur (Selbst-)Beobachtung angehalten werden.

Es wird empfohlen, Patienten nach Einleitung einer antipsychotischen Therapie in kurzen Abständen 
- wöchentlich während der ersten beiden Wochen nach Therapiebeginn oder Dosiserhöhung - auf Anzeichen von EPMS nachzubeobachten. Ist eine stabile Dosis erreicht, so ist eine Kontrolle auf Anzeichen einer TD in Abständen von 6 Monaten für Patienten, die mit FGA behandelt werden, und von 12 Monaten bei NGA indiziert. Bei Patienten mit hohem Risiko sind die Abstände zwischen den Kontrollen jeweils mindestens zu halbieren [42].

\section{Wahl des Antipsychotikums}

Bei der Wahl für einen individuellen Patienten sind Risiko und Nutzen einer Substanz einzuschätzen und während der Therapie regelmäßig zu überprüfen. Das TD-Risiko ist bei Verwendung von NGA geringer [43, 44]. Eine Untersuchung hat gezeigt, dass das Risiko für TD unter dem klassischen Antipsychotikum Haloperidol am höchsten ist [45]. Aber auch innerhalb der Klasse der NGA bestehen Unterschiede hinsichtlich des TD-Risikos. Laut Kenney et al. könnte ein Ranking bezüglich des TD-Risikos so aussehen: Clozapin < Quetiapin $<$ Aripiprazol $<$ Olanzapin = Ziprasidon $<$ Risperidon [43, 45-47].

Es sei noch einmal darauf hingewiesen, dass auch Medikamente, die nicht der Behandlung psychotischer Störungen dienen, TD verursachen können. So haben Kenney et al. in einer Untersuchung an einer Population, die sich in einer Bewegungsstörungsambulanz vorstellten, gezeigt, dass Metoclopramid das zweithäufigste TD-auslösende Medikament war [45]. Aufgrund des hohen, mit Metoclopramid assoziierten TD-Risikos hat die Europäische Arzneimittelbehörde (EMA) eine Empfehlung bezüglich einer Anwendungsbeschränkung dieser Substanz abgegeben, wonach die Anwendung nicht länger als 5 Tage bei einer täglichen Maximaldosierung von 3-mal $10 \mathrm{mg}$ erfolgen sollte [48].

\section{Behandlung der tardiven Dyskinesie}

Erster Schritt im Management der TD ist die Verifizierung der Diagnose. Abhängig von der psychiatrischen Grunderkrankung/Grunddiagnose ist, wenn dies möglich erscheint, das Absetzen der antipsychotischen Therapie oder eine Dosisreduktion in Erwägung zu ziehen. Für Patienten mit einer Langzeittherapie aufgrund von Schizophrenie oder bipolarer Störung sind Absetz- bzw. Dosisreduktionsversuche in der Regel keine Option. Bei diesen Patienten wird eine Umstellung der antipsychotischen Therapie auf eine Substanz mit geringerem Risiko für TD oder die symptomatische medikamentöse Therapie der TD empfohlen. In Reviews wird häufig die Umstellung auf Clozapin oder Quetiapin empfohlen [37, 49].

Die Datenlage bezüglich des Absetzens des Antipsychotikums ist uneinheitlich. So zeigte eine Studie an 49 psychiatrischen Patienten, von denen bei 36 das Antipsychotikum abgesetzt werden konnte, nur bei
$2 \%$ ein vollständiges Verschwinden der TD-Symptomatik und bei $20 \%$ eine Besserung [50]. Die retrospektive Analyse der Daten von 108 nicht schizophrenen Patienten hat ergeben, dass nach Absetzen der TDauslösenden Medikation bei $13 \%$ die TD nach 3 Jahren komplett verschwunden war [51]. Aufgrund dieser uneinheitlichen Datenlage kann ein Absetzen der antipsychotischen Therapie zur Behandlung der TD nicht eindeutig empfohlen werden [52]. Darüber hinaus kann das Absetzen eines Antipsychotikums die TD, zumindest initial für einige Wochen, verschlechtern $[53,54]$. So es die Grunderkrankung zulässt, kann ein Versuch, die Dosis der antipsychotischen Medikation zu reduzieren, erfolgen. Besonders ist darauf hinzuweisen, dass muskarinische Anticholinergika klassische (choreatische) tardive Dyskinesien verstärken können. Wichtig für die Behandlung der TD ist daher die Differenzierung zwischen „klassischer TD“ und tardiver Dystonie.

\section{Medikamentöse Therapie der „klassischen TD“}

Insgesamt sind bisher 175 Substanzen auf deren Wirksamkeit bei TD untersucht worden. Nach Meinung der Autoren liegt für die im Folgenden angeführten Substanzen die beste Evidenz vor.

\section{Amantadin}

Amantadin ist ein nichtkompetitiver NMDA-Rezeptorantagonist, der in kontrollierten und nichtkontrollierten Studien an Patienten mit TD untersucht wurde. Dabei konnte in einer doppelblinden, randomisierten Studie (RCT) im Vergleich zu Placebo unter Amantadin, das in Kombination mit einem Antipsychotikum verabreicht worden war, eine Reduktion im AIMS-Score (Abnormal Involuntary Movement Scale) um $15 \%$ erzielt werden [55]. In einer jüngeren RCT konnte mit Amantadin ein Rückgang der Dyskinesien um 21 \% erzielt werden, während in der Placebogruppe keine Verbesserung zu beobachten war [51]. Mögliche Nebenwirkungen von Amantadin beinhalten innere Unruhe, Verwirrtheitszustände und manchmal schmerzhafte Livedo reticularis.

\section{Clonazepam}

Die Verwendung des GABA-A-Agonisten Clonazepam in der Therapie der TD wurde in den 1970er- und 1980er-Jahren untersucht, was schließlich in der $\mathrm{Pu}-$ blikation einer RCT im Jahr 1990 resultierte [56]. Darin wurde bei 19 Patienten eine Reduktion um $37 \%$ im Dystoniescore der „Maryland Psychiatric Research Center Movement Disorder Scale“ erzielt. Bei 5 Patienten, die über bis zu 9 Monaten mit Clonazepam weiterbehandelt worden waren, ging die Wirkung nach 5-8 Monaten allerdings wieder verloren. Typische Nebenwirkungen waren Sedierung und Toleranzentwicklung. 


\section{Ginkgo biloba (EGb-761)}

Ginkgo biloba wurde in einer chinesischen Studie an 157 Patienten mit Schizophrenie und TD getestet [57]. Dabei konnte bei $53 \%$ der Patienten ein Therapieansprechen, definiert als zumindest 30 \%ige Reduktion im AIMS-Score, beobachtet werden. Dieser Effekt hielt zumindest über 6 Monate an. Ob Patienten auf die Therapie mit Ginkgo biloba ansprechen, ist vermutlich vom Vorhandensein eines Polymorphismus im BDNF-Gen abhängig [58]. Zu beachten ist das Interaktionspotenzial aufgrund der Enzyminduktion.

\section{Levetiracetam}

Dieses Antikonvulsivum führte in einer kleinen randomisierten Studie gegenüber Placebo zu einer 19 \%igen Reduktion des AIMS-Scores [59]. Als Nebenwirkung wurde Irritabilität und manchmal Parkinsonismus beobachtet.

\section{Tetrabenazin}

In insgesamt 11 Studien wurde die Wirksamkeit der Substanz an 403 Patienten mit TD geprüft [6]. In 9 dieser Studien kamen die Autoren zu dem Schluss, dass Tetrabenazin sicher und effektiv ist, sodass die American Academy of Neurology (AAN) Tetrabenazin in ihren Guidelines als Therapieoption empfiehlt [48]. $\mathrm{Zu}$ den typischen Nebenwirkungen zählen Sedierung, Parkinsonismus und depressive Symptome, wobei die Nebenwirkungen nach Dosisreduktion meist reversibel sind.

\section{Tiaprid}

Für Tiaprid liegt zwar keine Evidenz aus kontrollierten Studien zur Behandlung von TD vor, es handelt sich jedoch um die einzige in Österreich in der Indikation TD zugelassene Substanz.

\section{Vitamine E und B6}

Antioxidantien wie Vitamin E und Vitamin B6 wurden auch geprüft. Zu Vitamin E liegen widersprüchliche Ergebnisse vor. Während in einigen Studien Verbesserungen im Bereich zwischen 18,5\% und $43 \%$ beobachtet wurden, waren andere Studien negativ [48]. Auch für Vitamin B6 ist die Datenlage uneinheitlich.

\section{Andere Substanzen}

Andere Substanzen, die in kleinen Studien bzw. Kasuistiken Hinweise auf Wirksamkeit zeigten, sind Propranolol [60] und Piracetam [61].

\section{Symptomatische Behandlung der tardiven Dystonie}

Zur Therapie der tardiven Dystonie liegen keine Daten aus RCTs vor. Im Folgenden werden daher kursorisch die relativ bestuntersuchten Medikamente genannt. Dazu zählen Anticholinergika, Baclofen, Tetrabenazin und Clonazepam [48]. Bei fokalen tardiven Dystonien ist ein Therapieversuch mit Botulinumtoxin zu erwägen [48]. Bei therapierefraktären, schweren tardiven
Dystonien kann die tiefe Hirnstimulation des Globus pallidus internus eine Therapieoption darstellen, wobei das Verfahren der tiefen Hirnstimulation bei der tardiven Dystonie bisher ein experimentelles Verfahren darstellt [62].

\section{Limitationen}

Aufgrund der geringen Anzahl an methodisch sauberen Studien lassen sich keine eindeutigen Aussagen über die Wirksamkeit der Therapieoptionen treffen. Die Bewertung der verfügbaren Daten in Reviews und Metaanalysen gestaltet sich schwierig, da die Qualität der Studien sehr heterogen ist. Darüber hinaus besteht hinsichtlich der Patientenpopulationen große Inhomogenität betreffend Alter, Diagnose, Komorbiditäten und des auslösenden Antipsychotikums.

Open access funding provided by University of Innsbruck and Medical University of Innsbruck

Interessenkonflikt Die Erstellung dieses Manuskripts wurde von der Firma AOP Orphan logistisch unterstützt. Keiner der Autoren hat ein Honorar erhalten. Redaktionelle Unterstützung erfolgte durch Harald Leitner und Monika Kirchmayer von MEDahead. Prof. Fleischhacker hat Forschungsunterstützung von Janssen-Cilag, Otsuka und Lundbeck erhalten. Er hat Berater- und Vortragshonorare von AOP Orphan, Boehringer Ingelheim, Janssen, Richter, Roche, Lundbeck, Otsuka, Takeda, Amgen, Teva und Dainippon Sumitomo erhalten. Assoz.-Prof. PD Dr. Hofer hat Forschungsunterstützung von Janssen-Cilag, Berater- und Vortragshonorare von AOP Orphan, Janssen-Cilag, Lundbeck und Kongressunterstützung von Janssen-Cilag, Lundbeck, Sanofi Aventis erhalten. Prim. Dr. Jagsch hat Vortragshonorare von CSC Angelini, Unterstützungen für von ihm organisierte Fortbildungsveranstaltungen - Alterspsychiatrische Tagung 2016: Austroplant, AOP Orphan Pharmaceuticals, Germania, Gerot Lannacher Pharma, KRKA Pharma, Mundipharma und Kongresseinladung DGPPN: Pfizer erhalten. Univ.-Prof. Dr. Pirker erhielt in den letzten Jahren Reisekostenunterstützung bzw. Berater- und Vortragshonorare von Medtronic, Boehringer Ingelheim, Novartis, AbbVie, Lundbeck, AOP Orphan, UCB Pharma, Tewa, Ipsen Pharma, Licher MT und Actavis. Univ.Prof. Dr. Rittmannsberger hat Grants und Honorare von Janssen Cilag und Lundbeck erhalten. Univ.-Prof. Dr. Seppi hat Grants von Oesterreichische Nationalbank, FWF Austrian Science Fund, Michael J. Fox Foundation, International Parkinson and Movement Disorder Society und Honorare von Teva, UCB, Lundbeck, AOP Orphan Pharmaceuticals AG und International Parkinson and Movement Disorder Society erhalten. Chefarzt Prim. Dr. Psota gibt an, dass kein Interessenkonflikt besteht.

Open Access Dieser Artikel wird unter der Creative Commons Namensnennung 4.0 International Lizenz (http:// creativecommons.org/licenses/by/4.0/deed.de) veröffentlicht, welche die Nutzung, Vervielfältigung, Bearbeitung, Verbreitung und Wiedergabe in jeglichem Medium und Format erlaubt, sofern Sie den/die ursprünglichen Autor(en) und die Quelle ordnungsgemäß nennen, einen Link zur Creative Commons Lizenz beifügen und angeben, ob Änderungen vorgenommen wurden. 


\section{Literatur}

1. Sachdev P. The current status of tardive dyskinesia. Aust NZ JPsychiatry. 2000;34:355-69.

2. TarsyD, Baldessarini RJ.Epidemiology of tardivedyskinesia: is risk declining with modern antipsychotics? Mov Disord. 2006;21:589-98.

3. Sigwald J, Bouttier D, Raymondeaud C, Piot C. 4 Cases of facio-bucco-linguo-masticatory dyskinesis of prolonged development following treatment with neuroleptics. Rev Neurol (Paris) 1959; Jun;100:751-5

4. Faurbye A, Rasch PJ, Petersen PB, Brandborg G, Pakkenberg H.NEUROLOGICALSYMPTOMSINPHARMACOTHERAPY OF PSYCHOSES. Acta Psychiatr Scand. 1964; 40:10-27

5. American Psychiatric Association (APA): 2013; DSM-5

6. Aquino CCH, Lang AE. Tardive dyskinesia syndromes: current concepts. Parkinsonism Relat Disord. 2014;20(Suppl 1):113-7.

7. Bhidayasiri R, Truong DD. Chorea and related disorders. Postgrad Med J.2004;80:527-34.

8. Jankovic J. Tardive syndromes and other drug-induced movement disorders. Clin Neuropharmacol. 1995;18:197-214.

9. Bhidayasiri R, Bronstein JM. Improvement of cervical dystonia: possible role of transcranial magnetic stimulation simulating sensory tricks effect. Med Hypotheses. 2005;64:941-5.

10. Stacy M, Jankovic J. Tardive Dyskinesia. Curr Opin Neurol Neurosurg. 1991;4:343-9.

11. TarsyD, BaldessariniRJ.Epidemiology oftardivedyskinesia: is risk declining with modern antipsychotics? Mov Disord. 2006;21(5):589-98.

12. Eberhard J, Lindström E, Levander S. Tardive dyskinesia and antipsychotics: a 5-year longitudinal study of frequency, correlates and course. Int Clin Psychopharmacol. 2006;21:35-42.

13. Cavallaro R, Regazzetti MG, Mundo E, Brancato V, Smeraldi E. Tardive dyskinesia outcomes: clinical and pharmacologic correlates of remission and persistence. Neuropsychopharmacology. 1993;8:233-9.

14. Rittmannsberger $\mathrm{H}$. Ten year outcome of tardive dyskinesia during continuous treatment with first generation antipsychotics. Psychiatr Danub. 2008;20(4):461-5.

15. Kane JM. et al. Tardive Dyskinesia: a task force report of the American Psychiatric Association. Washington D.C.: American Psychiatric Association; 1992.

16. FleischhackerWW, WidschwendterC. Extrapyramidal sideeffects: avoidance, diagnosis and treatment. Psychiatr Prax. 2005;32(Suppl.1):25-30.

17. Margolese HC, Chouinard G, Kolivakis TT, Beauclair L, Miller R, Annable L. Tardive dyskinesia in the era of typical and atypical antipsychotics. Part 2: Incidence and management strategies in patients with schizophrenia. Can JPsychiatry. 2005;50:703-14.

18. Jeste DV. Tardive dyskinesia rates with atypical antipsychotics in older adults. J Clin Psychiatry. 2004;65(Suppl.9):21-4.

19. Kane JM. Tardive dyskinesia rates with atypical antipsychotics in adults: prevalence and incidence. J Clin Psychiatry. 2004;65(Suppl.9):16-20.

20. Bhidayasiri R. Boonyawairoj, Spectrum of tardive syndromes: clinical recognition and management. Postgrad Med J.2011;87:132-41.

21. Sethi KD, Morgan JC. Drug induced movement disorders. In: Jankovics JJ, Tolosa E (Hrsg.). Parkinson's disease and movement disorders. New York: Lippincott Williams \& Wilkins; 2007.S.394-408.
22. Correll CU, Schenk EM. Tardive dyskinesia and new antipsychotics. Curr Opin Psychiatry. 2008;21(2):151-6.

23. Winsberg BG, Hurwic MJ, Perel J. Neurochemistry of withdrawal emergent symptoms in children. Psychopharmacol Bull. 1977;13:38-40.

24. Klawans HL Jr., McKendall RR. Observations on the effect of levodopa on tardive lingual-facial-buccal dyskinesia. J Neurol Sci. 1971;14:189-92.

25. Klawans HL Jr., Rubovits R. An experimental model of tardive dyskinesia. J Neural Transm. 1972;33:235-46.

26. Marsden CD, Jenner P. The pathophysiology of extrapyramidal side-effects of neuroleptic drugs. Psychol Med. 1980;10:55-72.

27. Calabresi P, de Murtas M, Mercuri NB, Bernardi G. Chronic neuroleptic treatment: D2 dopamine receptor supersensitivity and striatal glutamatergic transmission. Ann Neurol. 1992;31:366-73.

28. Andreassen OA, Jorgensen HA. Neurotoxicity associated with neuroleptic-induced oral dyskinesias in rats. Implications for tardive dyskinesia? Prog Neurobiol. 2000;61:525-41.

29. Burger M, Fachinetto R, Calegari L, Paixão MW, Braga AL, Rocha JB. Effects of age on reserpine-induced orofacial dyskinesia and possible protection of diphenyl diselenide. Brain Res Bull. 2004;64:339-45.

30. Miller R, Chouinard G. Loss of striatal cholinergic neurons as a basis for tardive and L-dopa-induced dyskinesias, neuroleptic-induced supersensitivity psychosis and refractory schizophrenia. Biol Psychiatry. 1993;34:713-38.

31. Basile VS, Ozdemir V, Masellis M, Walker ML, Meltzer HY, Lieberman JA, Potkin SG, Alva G, Kalow W, Macciardi FM, Kennedy JL. A functional polymorphism of the cytochrome P450 1A2 (CYP1A2) gene: association with tardive dyskinesia in schizophrenia. Mol Psychiatry. 2000;5:410-7.

32. Fu Y, Fan CH, Deng HH, Hu SH, Lv DP, Li LH, Wang JJ, Lu XQ. Association of CYP2D6 and CYP1A2 gene polymorphism with tardive dyskinesia in Chinese schizophrenic patients. Acta Pharmacol Sin. 2006;27:328-32.

33. Tiwari AK, Deshpande SN, Rao AR, Bhatia T, Mukit SR, Shriharsh V, Lerer B, Nimagaonkar VL, Thelma BK. Genetic susceptibility to tardive dyskinesia in chronic schizophrenia subjects: I. Association of CYP1A2 gene polymorphism. Pharmacogenomics J. 2005;5:60-9.

34. Teo JT, Edwards MJ, Bhatia K. Tardive dyskinesia is caused by maladaptive synaptic plasticity: a hypothesis. Mov Disord. 2012;27(10):1205-15.

35. Andreassen OA, Finsen B, Ostergaard K, Sørensen JC, West MJ, Jørgensen HA. The relationship between oral dyskinesias produced by long-term haloperidol treatment, the density of striatal preproenkephalin messenger RNA and enkephalin peptide, and the number of striatal neurons expressing preproenkephalin messenger RNA in rats. Neuroscience. 1999;88:27-35.

36. Gunne LM, Andren PE. An animal model for coexisting tardive dyskinesia and tardive parkinsonism: a glutamate hypothesis for tardive dyskinesia. Clin Neuropharmacol. 1993;16:90-5.

37. Cloud JL, Zutshi D, Factor SA. Tardive dyskinesia: therapeutic options for an increasingly common disorder. Neurotherapeutics. 2014;11(1):166-76.

38. Glazer WM, Morgenstern H, Schooler N, Berkman CS, Moore DC. Predictors of improvement in tardive dyskinesia following discontinuation of neuroleptic medication. Br J Psychiatry. 1990;157:585-92.

39. AMG $\$ 8$ Abs. 1; BGBI 1983/185idF BGBII $2005 / 153$

40. World Medical Association. WMA declaration of Helsink - ethical principles for medical research involving hu- 
man subjects. http://www.wma.net/en/30publications / 10policies/b3/index.html.Zugegriffen:23. Nov 2015.

41. Tenback DE, van Harten PN, van Os J. Non-therapeutic risk factors for onset of tardive dyskinesia in schizophrenia: a meta-analysis. Mov Disord.2009;24(16):2309-15.

42. Marder SR, Essock SM, Miller AL, Buchanan RW, Casey DE, Davis JM, Kane JM, Lieberman JA, Schooler NR, Covell N, StroupS, Weissman EM, Wirshing DA, Hall CS, Pogach L, PiSunyer X, Bigger JT Jr, Friedman A, Kleinberg D, Yevich SJ, Davis B, Shon S. Physical health monitoring of patients with schizophrenia. Am J Psychiatry. 2004;161(8):1334-49.

43. Correll CU, Leucht S, Kane JM. Lower risk for tardive dyskinesia associated with second-generation antipsychotics: a systematic review of 1-year studies. Am J Psychiatry. 2004;161:414-25.

44. Tenback DE, van Harten PN, Slooff CJ, Belger MA, van Os J, SOHO Study Group. Effects of antipsychotic treatment on tardive dyskinesia: a 6-month evaluation of patients from the European Schizophrenia Outpatient Health Outcomes (SOHO) Study. JClin Psychiatry. 2005;66:1130-3.

45. Kenney C, Hunter C, Davidson A, Jankovic J. Metoclopramide, an increasingly recognized cause of tardive dyskinesia. JClin Pharmacol. 2008;48:379-84.

46. Nasrallah HA. Focus on lower risk of tardive dyskinesia with atypical antipsychotics. AnnClin Psychiatry. 2006;18:57-62.

47. Friedmann JH. Atypical antipsychotics in the EPS-vulnerable patient. Psychoneuroendocinology. 2003;28(suppl 1):39-51.

48. EMA2013:Article31 of Directive2001/83/EC

49. Saifee TA, Edwards MJ. Tardive movement disorders: a practical approach. Pract Neurol.2011;11(6):341-8.

50. Glazer WM, Morgenstern H, Schooler N, Berkman CS, Moore DC. Predictors of improvement in tardive dyskinesia following discontinuation of neuroleptic medication. Br J Psychiatry. 1990;157:585-92.

51. Pappa S, Tsouli S, Apostolou G, Mavreas V, Konitsiotis S. Effects of amantadine on tardive dyskinesia: a randomized, double-blind, placebo-controlled study. Clin Neuropharmacol. 2010;33:271-5.

52. Bhidayasiri R, Fahn S, Weiner WJ, Gronseth GS, Sullivan KL, Zesiewicz TA, American Academy of Neurology. Evidencebased guideline: treatment of tardive syndromes: report of
theGuidelineDevelopmentSubcommittee of theAmerican Academy of Neurology. Neurology. 2013;81:463-9.

53. Gardos G, Cole JO, Rapkin RM, LaBrie RA, Baquelod E, Moore P, Sovner R, Doyle J. Anticholinergic challenge and neuroleptic withdrawal. Changes in dyskinesia and symptom measures. Arch Gen Psychiatry. 1984;41:1030-5.

54. Branchey MH, Branehey LB, Richardson MA. Effects of gradual decrease and discontinuation of neuroleptics on clinical condition and tardive dyskinesia [proceedings]. Psychopharmacol Bull. 1981;17:118-20.

55. Angus S, Sugars J, Boltezar R, Koskewich S, Schneider NM. A controlled trial of amantadine hydrochloride and neuroleptics in the treatment of tardive dyskinesia. J Clin Psychopharmacol. 1997;17:88-91.

56. Thaker GK, Nguyen JA, Strauss ME, Jacobson R, Kaup BA, Tamminga CA. Clonazepam treatment of tardive dyskinesia: a practical GABAmimetic strategy. Am J Psychiatry. 1990;147:445-51.

57. Zhang WF, Tan YL, Zhang XY, Chan RC, Wu HR, Zhou DF. Extract of Ginkgo biloba treatment for tardive dyskinesia in schizophrenia: a randomized, double-blind, placebocontrolled trial. J Clin Psychiatry. 2011;72:615-21.

58. Zhang XY, Zhang WF, Zhou DF, da Chen C, Xiu MH, Wu HR, Haile CN, Kosten TA, Kosten TR. Brain-derived neurotrophic factor levels and its Val66Met gene polymorphism predict tardive dyskinesia treatment response to Ginkgo biloba. Biol Psychiatry. 2012;72(8):700-6.

59. Woods SW, Saksa JR, Baker CB, Cohen SJ, Tek C. Effects of levetiracetam on tardive dyskinesia: a randomized, double-blind, placebo-controlled study. J Clin Psychiatry. 2008;69(4):546-54.

60. Factor SA. Propranolol therapy for tardive dyskinesia revisited. MovDisord. 2012;27(13):1703.

61. Libov I, Miodownik C, Bersudsky Y, Dwolatzky T, Lerner V. Efficacy of piracetam in the treatment of tardive dyskinesia in schizophrenic patients: a randomized, double-blind, placebo-controlled crossover study. J Clin Psychiatry. 2007;68(7):1031-7.

62. Mentzel CL, Tenback DE, Tijssen MA, Visser-Vandewalle VE, van Harten PN. Efficacy and safety of deep brain stimulation in patients with medication-induced tardive dyskinesia and/or dystonia: a systematic review. J Clin Psychiatry. 2012;73(11):1434-8. 